Check for updates

Cite this: RSC Adv., 2018, 8, 34476

\title{
Mechanism of sulfidation of small zinc oxide nanoparticles
}

\author{
Progna Banerjee (D) a and Prashant K. Jain (D) *abcd
}

$\mathrm{ZnO}$ has industrial utility as a solid sorbent for the removal of polluting sulfur compounds from petroleum-based fuels. Small ZnO nanoparticles may be more effective in terms of sorption capacity and ease of sulfidation as compared to bulk $\mathrm{ZnO}$. Motivated by this promise, here, we study the sulfidation of ZnO NPs and uncover the solid-state mechanism of the process by crystallographic and optical absorbance characterization. The wurtzite-structure ZnO NPs undergo complete sulfidation to yield ZnS NPs with a drastically different zincblende structure. However, in the early stages, the ZnO NP lattice undergoes only substitutional doping by sulfur, while retaining its wurtzite structure. Above a threshold sulfur-doping level of $30 \mathrm{~mol} \%$, separate zincblende ZnS grains nucleate, which grow at the expense of the ZnO NPs, finally yielding ZnS NPs. Thus, the full oxide to sulfide transformation cannot be viewed simply as a topotactic place-exchange of anions. The product ZnS NPs formed by nucleation-growth share neither the crystallographic structure nor the size of the initial ZnO NPs. The reaction mechanism may inform the future design of nanostructured $\mathrm{ZnO}$ sorbents.

Received 20th August 2018

Accepted 4th October 2018

DOI: $10.1039 / \mathrm{c} 8 \mathrm{ra06949b}$

rsc.li/rsc-advances the manner in which these NPs undergo sulfidation. The structural mechanism of the sulfidation process ${ }^{30}$ may have critical differences compared to bulk $\mathrm{ZnO}$ powders or even larger NPs of tens of $\mathrm{nm}$ in $\operatorname{size}^{24}$ and may therefore influence sorbent design. In a seminal study, Park et $a l^{30}$ studied the sulfidation of hexagonal-shaped $14 \mathrm{~nm}$ ZnO nanocrystals (NCs) at high temperature $\left(235^{\circ} \mathrm{C}\right)$ using hexamethyldisilathiane. The reaction was found to involve the anion exchange of $\mathrm{O}^{2-}$ with $\mathrm{S}^{2-}$ in the NC lattice. The overall shape and crystallography of ZnS NCs was templated by the initial ZnO NCs. However, due to the faster outward diffusion of $\mathrm{Zn}^{2+}$ as compared to the inward diffusion of $\mathrm{S}^{2-}$, the exchange reaction was accompanied by a nanoscale Kirkendall phenomenon, as a result of which the ZnS NCs formed were hollow.

Here, we track the step-wise sulfidation of smaller (ca. $5 \mathrm{~nm}$ ) ZnO NPs using optical spectroscopy and X-ray crystallography. Prior to the onset of sulfidation, $\mathrm{O}^{2-}$ in wurtzite $\mathrm{ZnO}$ NPs undergoes substitutional doping with $\mathrm{S}^{2-}$ without any major change in its structure. Upon reaching a critical concentration of sulfur doping, separate zincblende $\mathrm{ZnS}$ grains form and grow into ZnS NPs. Thus, the sulfidation of these small ZnO NPS studied here is not simply a topotactic or templated placeexchange of anions; rather the nucleation and growth of a separate $\mathrm{ZnS}$ crystallite is involved in the latter stages.

\section{Experimental methods}

\section{Synthesis of ZnO NPs}

The synthesis of $4 \mathrm{~nm}$ ZnO NPs was based on a procedure from Kamat and coworkers ${ }^{31}$ with some changes. Briefly, $0.05 \mathrm{M}$ of zinc acetate dihydrate $(1.095 \mathrm{~g})$ was added to $50 \mathrm{~mL}$ of ethanol 
in a $100 \mathrm{~mL}$ three-necked round-bottom flask. The flask was put under $\mathrm{Ar}$, heated to $80{ }^{\circ} \mathrm{C}$, and refluxed for $2 \mathrm{~h}$ followed by gradual cooling down to room-temperature over the course of $1.5 \mathrm{~h}$. The zinc precursor solution $(0.05 \mathrm{M})$ was stored in a desiccator and was used for a period of up to three months from the preparation date. Whenever needed, a batch of $\mathrm{ZnO}$ NP colloid was prepared by the hydrolysis of the zinc precursor using lithium hydroxide ( $\mathrm{LiOH})$ powder. Typically, $20 \mathrm{mg}$ of $\mathrm{LiOH}$ was added directly to $3 \mathrm{~mL}$ of the precursor solution diluted with $12 \mathrm{~mL}$ of ethanol. The resulting reaction mixture was sonicated for $20 \mathrm{~min}$ without heating. The resulting colloid solution was washed by adding an equal volume of acetone and centrifuging at $10000 \mathrm{rpm}$ for $20 \mathrm{~min}$. The precipitated colloid was redispersed in $25 \mathrm{~mL}$ of ethanol. The colloid was allowed to stand for $5 \mathrm{~h}$ or more to allow NP growth to stabilize before sulfidation studies or other measurements. The $\mathrm{ZnO}$ colloid was typically used up to a week from its preparation.

\section{Sulfidation of ZnO NPs}

The conversion from $\mathrm{ZnO}$ to $\mathrm{ZnS}$ NPs was carried out at a temperature of $45{ }^{\circ} \mathrm{C}$. We started with a colloid of ZnO NPs dispersed in ethanol (at a concentration which yields an absorbance of $\sim 1$ at the $\mathrm{ZnO}$ excitonic peak maximum). The colloid was transferred to a $20 \mathrm{~mL}$ glass vial immersed in a water bath set at $45{ }^{\circ} \mathrm{C}$. To this colloid, a solution of sodium sulfide $\left(\mathrm{Na}_{2} \mathrm{~S}\right)$ in ethanol $\left(3.1 \mathrm{mg} \mathrm{mL}^{-1}\right)$ was added in aliquots of $10 \mu \mathrm{L}$ with vigorous stirring. After each addition, the reaction was allowed to approach completion by waiting a duration of $40 \mathrm{~min}$, after which $\sim 3.5 \mathrm{~mL}$ of the colloid was transferred to a quartz spectrophotometry cuvette and a UV-vis extinction spectrum in the range of 220-600 $\mathrm{nm}$ was measured. After the measurement, the colloid was returned to the reaction vial. The reaction appeared to reach saturation upon the addition of 11.9 $\mu \mathrm{mol}$ of $\mathrm{S}^{2-}$, at which point no further change in the excitonic features in the absorbance spectrum were observed.

At various stages of sulfidation, the NPs were subject to other characterization described below. For this, the colloid subject was subject to sulfidation in steps, as described above, up to a specific stage identified by the total molar amount of $\mathrm{S}^{2-}$ added. The colloid was then purified from excess reagents and salts by washing with acetone and centrifugation at $10000 \mathrm{rpm}$ for $20 \mathrm{~min}$. The precipitated colloid was redispersed in ethanol for further characterization.

\section{pXRD}

Powder X-ray diffraction (pXRD) patterns were collected for NPs at multiple stages of sulfidation. The diffraction measurements were conducted on a Rigaku Miniflex 600 powder X-ray diffractometer operated at full power ( $40 \mathrm{kV}$ to $15 \mathrm{~mA}$ ) with $\mathrm{Cu} \mathrm{K} \mathrm{K}_{\alpha}$ radiation wavelength $(1.54 \AA)$. Data was collected in reflection mode in the $2 \theta$ range of $20^{\circ}-75^{\circ}$ using a step size of $0.04^{\circ}$, with scan times lasting for $2-3 \mathrm{~h}$. pXRD samples were prepared by drop-casting NPs from a colloid dispersed in ethanol into a thick film on a zero-background quartz substrate. Before each run, the quartz substrate was cleaned by rinsing in aqua regia and DI water. Diffraction patterns were plotted in
Origin Lab software and manual baseline subtraction was performed for each of the patterns.

\section{Elemental analysis}

The elemental composition of the NPs, specifically the molar ratio of $\mathrm{S}: \mathrm{Zn}$, was determined at three stages: the initial $\mathrm{ZnO}$ NPs before sulfidation, the intermediate stage obtained by the addition of $1.99 \mu \mathrm{mol}$ of $\mathrm{S}^{2-}$, and the final ZnS NPs achieved by the addition of $11.9 \mu \mathrm{mol}$ of $\mathrm{S}^{2-}$ to the ZnO NPs. These samples were each purified of reagents and salts twice by washing with acetone and centrifugation. The washed precipitate was subsequently dried in quartz digestion tubes by flowing $\mathrm{N}_{2}$ gas. Samples were subject to inductively-coupled plasma atomic emission spectroscopy (ICP-AES) measurements, the results of which are presented in Table 1.

\section{Electron microscopy}

The initial ZnO NPs and final ZnS NPs were subject to TEM imaging. For $\mathrm{ZnO} \mathrm{NPs}, 6 \mathrm{~mL}$ of $\mathrm{ZnO}$ colloid prepared as described above was treated with $0.3 \mathrm{~mL}$ of oleic acid and washed with $6 \mathrm{~mL}$ of acetone followed by centrifugation at $10000 \mathrm{rpm}$ for $20 \mathrm{~min}$. Then the precipitated colloid was dispersed in ethanol. About $50 \mu \mathrm{L}$ of the diluted $\mathrm{ZnO}$ NP colloid was drop cast onto an ultrathin carbon grid, which was then allowed to dry in a vacuum desiccator. ZnS NPs were prepared by complete sulfidation of $6 \mathrm{~mL}$ of $\mathrm{ZnO}$ colloid accomplished, as described above, by addition of $\mathrm{S}^{2-}$. The $\mathrm{ZnS}$ NP colloid was treated with $0.3 \mathrm{~mL}$ of oleic acid and washed with $6 \mathrm{~mL}$ of acetone followed by centrifugation at $10000 \mathrm{rpm}$ for $20 \mathrm{~min}$. Then the precipitated colloid was dispersed in ethanol. The TEM grid, held by tweezers was dipped into the colloid once and then allowed to dry in a vacuum desiccator. TEM imaging was performed on a JEOL 2010-EFFEG instrument operating at 200 $\mathrm{kV}$. NP size analysis was performed using the Fiji software. The diameter of each NP identified in a representative image was determined manually along three arbitrary directions across the NP and averaged.

\section{Structure factor simulations}

For the initial ZnO and the early-stage sulfur-doped ZnO NCs, pXRD patterns were simulated using the program PowderCell. The program performs a structure factor calculation using crystal symmetry, lattice parameters, atomic positions, site occupancies and Debye-Scherer broadening as input parameters. Input parameters for each simulated pattern are presented in Table 2. The X-ray wavelength was set to $1.54 \AA$ corresponding to $\mathrm{Cu} \mathrm{K}_{\alpha 1}$ radiation. The starting model was $\mathrm{ZnO}$ in a wurtzite

Table 1 Elemental composition of the NPs at key stages in the sulfidation

\section{Stage} $\mathrm{S} / \mathrm{Zn}$, molar ratio

Initial (ZnO)

Intermediate $\left(1.99 \mu \mathrm{mol}\right.$ of $\mathrm{S}^{2-}$ added $)$ Final (ZnS) 
Table 2 Crystallographic parameters for structure factor simulations shown in Fig. $2 \mathrm{~b}$ and d

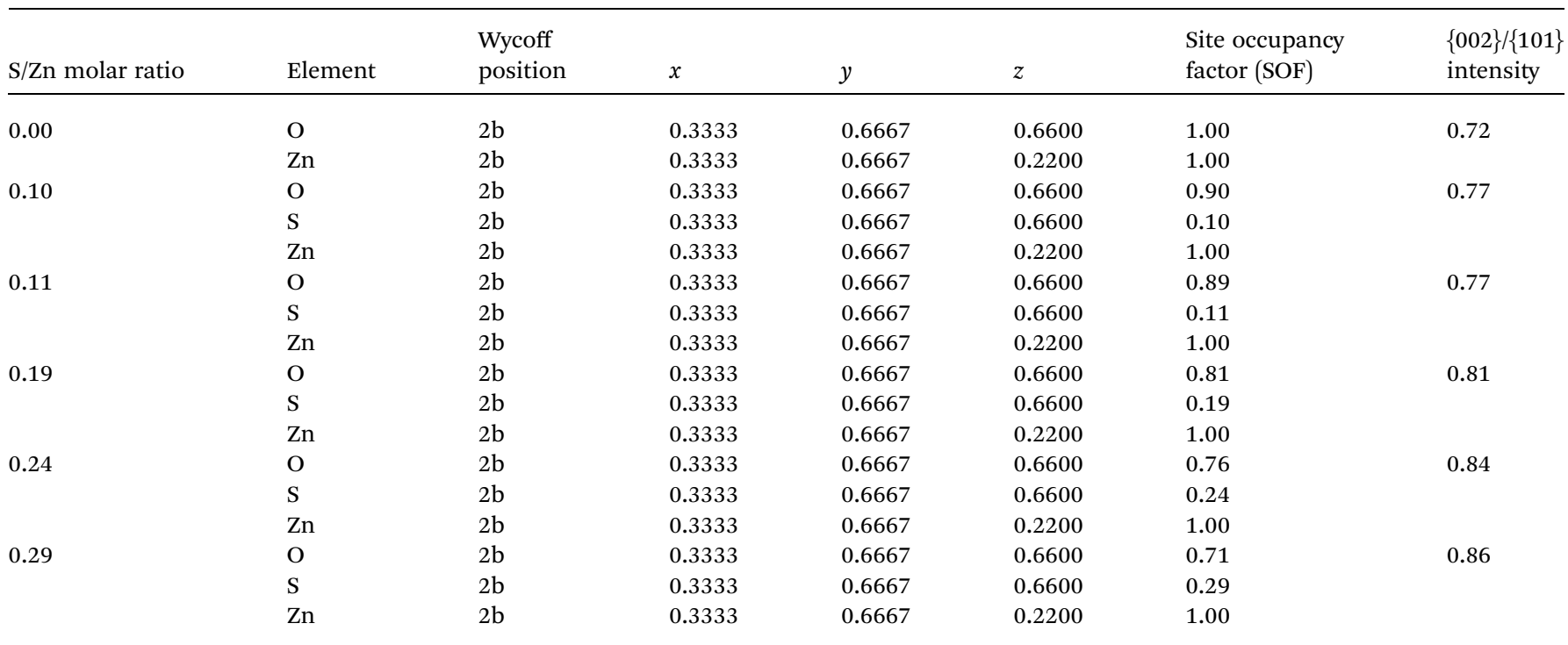

lattice, characterized by the space group $P 63 \mathrm{mmc}$. The $\mathrm{O}$ and the $\mathrm{Zn}$ atoms were placed in the $2 \mathrm{~b}$ Wycoff positions. The lattice parameters were set to $3.247 \AA$ and 5.179 A along the $a$ and the $c$ crystallographic directions, respectively, which provided the closest fit to the peak positions of the experimentally measured pXRD reflections of the wurtzite ZnO NPs. These lattice parameters, while close to the known parameters of bulk $\mathrm{ZnO}$ ( $a$ $=3.250 \AA$ and $c=5.204 \AA),{ }^{32}$ are marginally smaller, which may be explained by a small degree of compressive strain in the NPs. A peak-dependent Debye-Scherer broadening (FWHM) of $0.79^{\circ}$ was applied at a $2 \theta$ of $20^{\circ}$, along with a Lorentzian peak profile. The pXRD pattern simulated with these parameters matched the experimental pattern of the initial ZnO NPs, especially in terms of the $\{002\}:\{101\}$ peak intensity ratio. For sulfur-doped ZnO NCs, the structure was modeled by substitution of a fraction of the $\mathrm{O}^{2-}$ by $\mathrm{S}^{2-}$ in the $2 \mathrm{~b}$ Wycoff positions. Four different $\mathrm{S} / \mathrm{Zn}$ molar ratios of $0.1,0.11,0.24$, and 0.29 were tested, simulated pXRD patterns of which matched experimental patterns (in terms of the $\{002\}:\{101\}$ peak intensity ratio) of the NPs at added $\mathrm{S}^{2-}$ amount of $0.4,0.79,1.99$, and $2.78 \mu \mathrm{mol}$, respectively.

\section{Results and discusions}

The ZnO NPs, synthesized by a known procedure, ${ }^{31}$ had an average size of $4.7 \mathrm{~nm}$ (Fig. 1a and b) as found from TEM and a wurtzite structure as shown by their pXRD pattern (Fig. 2a, dark purple curve). The NPs were subject to sulfidation with $\mathrm{Na}_{2} \mathrm{~S}$ in ethanol at $45{ }^{\circ} \mathrm{C}$. The sulfidation was performed as a titration, wherein molar aliquots of $\mathrm{Na}_{2} \mathrm{~S}$ were added in a stepwise manner. Upon cumulative addition of $11.9 \mu \mathrm{mol}$ of $\mathrm{Na}_{2} \mathrm{~S}$ or $\mathrm{S}^{2-}$, the ZnO NPs underwent complete sulfidation. This is evidenced by the complete loss of diffraction peaks of wurtzite $\mathrm{ZnO}$ and the appearance of the $\{111\}$ and $\{220\}$ reflections of zincblende ZnS in the pXRD pattern (Fig. 2a, brown curve). In the elemental analysis (Table 1) of the final-stage NPs, a S/Zn molar of $c a .1$ was measured, which supports the complete sulfidation of ZnO NPs to form ZnS. TEM showed ZnS NPs with an average size of $2.7 \mathrm{~nm}$, significantly smaller than the size of the starting $\mathrm{ZnO}$ NPs. The smaller grain size of the $\mathrm{ZnS}$ is also reflected in the greater Debye-Scherer broadening of the pXRD peaks of the final-stage ZnS NPs (Fig. 2a, brown curve) as compared to those of the starting ZnO NPs (Fig. 2a, dark purple curve). From the Debye-Scherer broadening of the $\{111\}$ peak $\left(2 \theta=29^{\circ}\right.$, FWHM $=3.79^{\circ}$ ) of the final ZnS NPs, we estimated the NP size $D$ :

$$
D=\frac{0.94 \lambda}{\mathrm{FWHM} \text { in radians } \times \cos \theta}
$$

to be $22.6 \AA$ or $2.3 \mathrm{~nm}$. $\lambda$ is the incident X-ray wavelength of 1.54 $\AA$. The size estimated from pXRD is close to the one estimated by TEM (Fig. 1d).

At each step of the titration, the NP colloid was characterized by UV-vis extinction spectrophotometry (Fig. 1e) and pXRD (Fig. 2a), which shed light on the manner of sulfidation. The starting ZnO NPs exhibited an extinction spectrum with an exciton peak around $3.66 \mathrm{eV}$. This excitonic peak reflects the band-gap of these NPs, which is smaller than the bulk value ${ }^{33}$ of $3.37 \mathrm{eV}$ due to the effect of quantum confinement.

With the sequential addition of $\mathrm{S}^{2-}$ in the range of $0-1.99$ $\mu \mathrm{mol}$, the ZnO exitonic peak shifted to lower energies (Fig. 1f). This shift cannot be attributed to the formation of $\mathrm{ZnS}$. ZnS has a higher band-gap than $\mathrm{ZnO},{ }^{34}$ so $\mathrm{ZnS}$ formation would have resulted in a shift to higher energies. Moreover, pXRD showed that, in the $0-1.99 \mu \mathrm{mol}$ range of added $S^{2-}$, the NPs maintain their wurtzite ZnO structure (Fig. 2a) and no diffraction peaks related to ZnS are observed. However, elemental analysis of the NPs obtained by treatment with $1.99 \mu \mathrm{mol}$ of $\mathrm{S}^{2-}$ revealed a $\mathrm{S} / \mathrm{Zn}$ molar ratio of 0.27 (Table 1). Thus, the ZnO NPs appear to be significantly sulfur-doped. Such sulfur doping can explain the red-shift of the exciton peak. Zinc oxysulfides, $\mathrm{ZnO}_{1-x} \mathrm{~S}_{x}$, with $x$ $=0-0.3$ are known to have a lower band gap than $\mathrm{ZnO}$ due to the effect of band-bowing. ${ }^{34}$ If the sulfur-doping were to be limited to an outer shell of each NP, the resulting intermediate-stage NPs would consist of a shrunken $\mathrm{ZnO}$ core surrounded by 
(a)

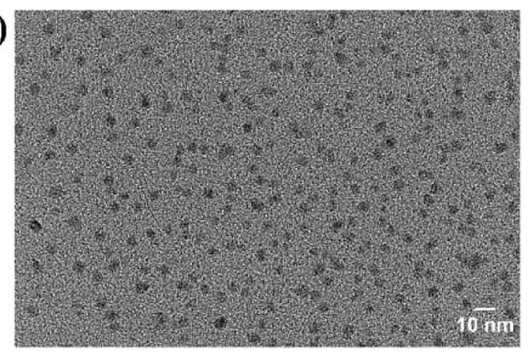

(c)

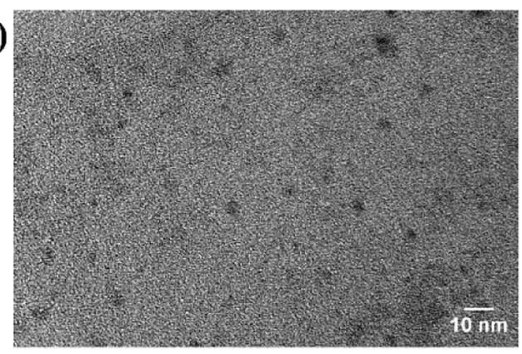

(e)

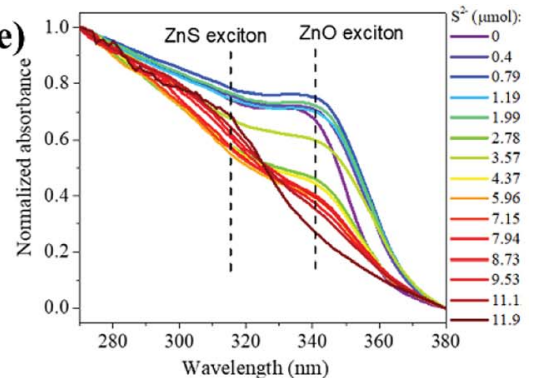

(b)

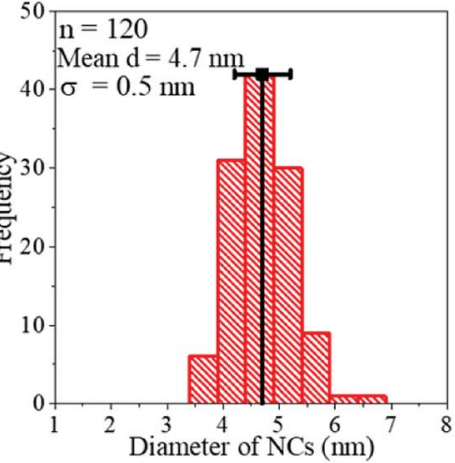

(d)

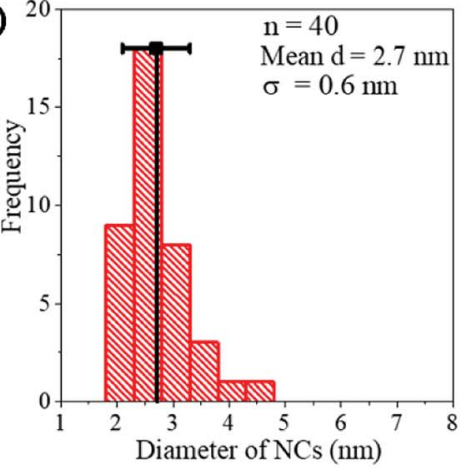

(f)

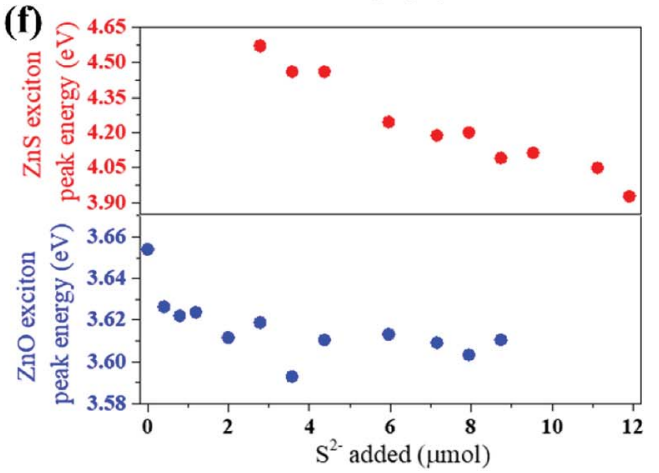

Fig. 1 (a) Representative TEM image of the synthesized ZnO NPs, (b) histogram of NP diameter for $n=120$ NPs showing an average of $4.7 \mathrm{~nm}$ with a standard deviation $(\sigma)$ of $0.5 \mathrm{~nm}$. (c) Representative TEM image of the synthesized ZnS NPs, (d) histogram of NP diameter for $n=40$ NPs showing an average of $2.7 \mathrm{~nm}$ with a standard deviation $(\sigma)$ of $0.6 \mathrm{~nm}$. (e) Absorbance spectra (normalized from 0 to 1 in the $270-380 \mathrm{~nm}$ range) of the colloid at various stages of $\mathrm{ZnO} N P$ sulfidation, in terms of the moles of $\mathrm{Na}_{2} \mathrm{~S}$ added. The exciton peak positions of the initial ZnO NPs and the final $Z n S \mathrm{NPs}$ are marked by the vertical dotted lines. (f) The $\mathrm{ZnO} N P$ and the $\mathrm{ZnS} \mathrm{NP}$ exciton peak energies in eV plotted as a function of the moles of $\mathrm{Na}_{2} \mathrm{~S}$ added. The exciton peak energies were extracted from the absorbance spectra in (e), which were first subject to manual baseline subtraction, without which it is difficult to discern from the plotted spectra the broad and weak ZnS exciton peak at the early stages of sulfidation.

a ZnS shell. Such NPs would exhibit a blue-shifted ZnO exciton peak as compared to the exciton peak of the initial ZnO NPs. On the other hand, we observe a red-shift of the ZnO exciton peak at the early stages in the sulfidation (Fig. 1f, bottom panel), which is consistent with the attainment of an oxysulfide composition. ${ }^{34}$ In other words, the sulfur-doping extends across the NP lattice.

Further confirmation of and insight into the sulfur-doping of the ZnO NPs at the early stages of the sulfidation $(0-2.78 \mu \mathrm{mol}$ of $\mathrm{S}^{2-}$ ) is obtained from a closer analysis of the pXRD findings. We performed pXRD simulations (Fig. 2d) of sulfur-doped ZnO lattices, where a fraction of the $\mathrm{O}^{2-}$ anions were replaced by $\mathrm{S}^{2-}$, without any other modification of the wurtzite structure. The simulated pXRD patterns of sulfur-doped $\mathrm{ZnO}$ resemble the simulated pattern for wurtzite $\mathrm{ZnO}$ (Fig. 2d). However, there is one difference: the ratio of the $\{002\}:\{111\}$ peak intensity is higher for the sulfur-doped ZnO NPs as compared to the undoped ZnO NPs. Larger the S/Zn molar ratio (Fig. 2d), greater is the ratio of the $\{002\}:\{111\}$ peak intensity. This computed trend matches with the trend observed in the experimental pXRD, as shown in Fig. 2b. Thus, the pXRD analysis confirms that at the early stages of the sulfidation, the ZnO NPs undergo substitutional doping by sulfur. Simulations predict that NPs subject to $1.99 \mu \mathrm{mol} \mathrm{S}{ }^{2-}$ have a S/Zn molar ratio of 0.24 , which is close to the elemental analysis findings (Table 1 ).

At the point of $2.78 \mu \mathrm{mol}$ added $\mathrm{S}^{2-}$, a broad absorption peak emerged at higher energies, i.e., $4.6 \mathrm{eV}$. This peak can be attributed to newly formed ZnS NPs. After all, ZnS is known to have a higher band-gap than ZnO. ${ }^{34}$ Upon further addition of $\mathrm{S}^{2-}$, the newly emerged excitonic peak increased in absorbance and shifted to lower energies (Fig. 1e and f). Alongside, the $\mathrm{ZnO}$ exciton peak decreased in strength until it was fully extinguished. These spectral trends can be explained by the following manner of sulfidation.

A small ZnS domain nucleates from the heavily sulfur-doped ZnO NPs. The considerably higher excitonic peak energy of $4.6 \mathrm{eV}$ relative to the bulk $\mathrm{ZnO}$ band-gap ${ }^{35}$ of $3.72 \mathrm{eV}$ suggests that the nucleated domain is ultrasmall. As more $\mathrm{S}^{2-}$ becomes available, the $\mathrm{ZnS}$ domains grow further, which is manifested in the red-shift of the ZnS excitonic peak and increase in its absorbance (Fig. 1e and f). Concomitantly, the ZnO domains, which serve as the source of $\mathrm{Zn}$, shrink in volume, which is reflected in a small blue-shift of the $\mathrm{ZnO}$ excitonic peak along with a decrease in its absorbance beyond $3.57 \mu \mathrm{mol}$ added $\mathrm{S}^{2-}$. Eventually, the ZnO excitonic peak was fully extinguished. The final ZnS NPs exhibited a spectrum with a single excitonic peak at $3.94 \mathrm{eV}$, corresponding to small NPs.

The pXRD results provide further support to the abovedescribed mechanism of sulfidation in the post-doping stages. At $4.37 \mu \mathrm{mol}$ of added $\mathrm{S}^{2-}$, a broad peak appeared at the position corresponding to the major $\{111\}$ reflection of zincblende $\mathrm{ZnS}$, which serves as a structural indicator of $\mathrm{ZnS}$ nucleation. This diffraction peak grew in relative intensity and also narrowed with increasing addition of $\mathrm{S}^{2-}$, which would be consistent with a growth in ZnS domains. Alongside, with increasing addition of $\mathrm{S}^{2-}$, the wurtzite $\mathrm{ZnO}$ diffraction peaks became 
(a)

(c)
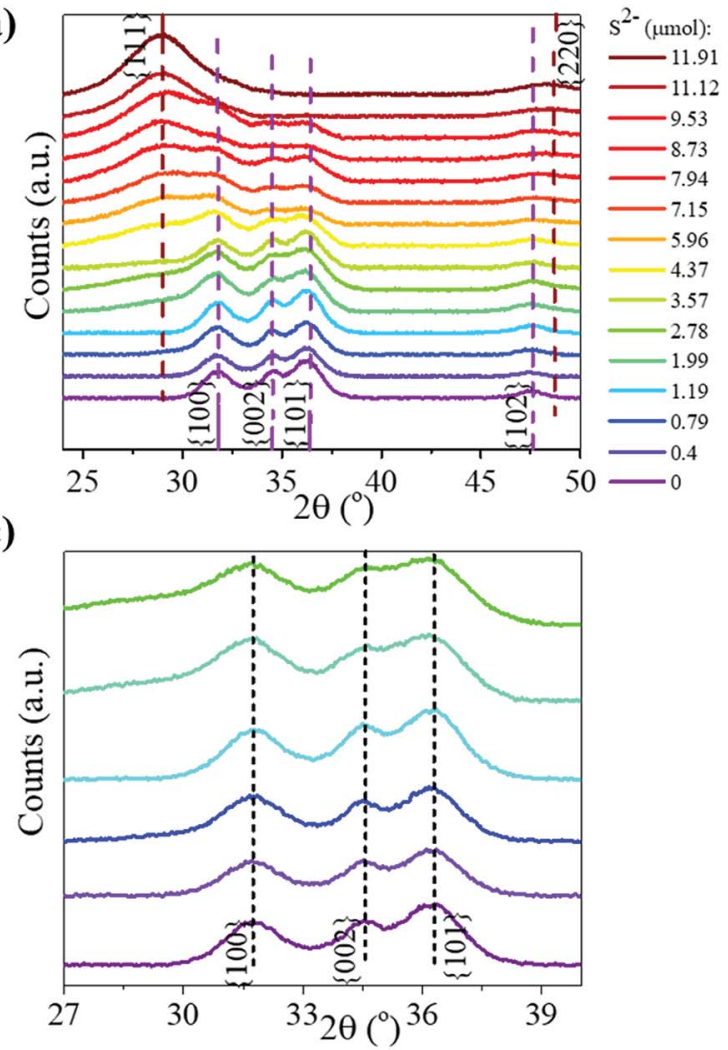

(b)

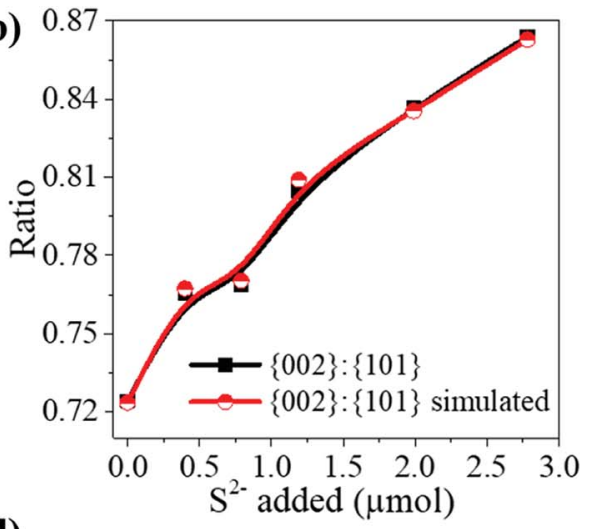

(d)

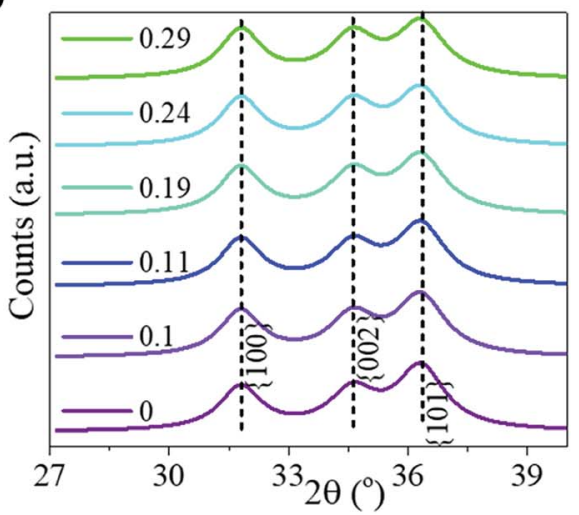

Fig. 2 (a) Experimental pXRD patterns (baseline subtracted) are shown for NPs at various stages of the sulfidation transformation from wurtzite $\mathrm{ZnO}$ to zincblende $\mathrm{ZnS}$, indicated by the moles of $\mathrm{Na}_{2} \mathrm{~S}$ added. Peaks are assigned to major reflections, as labeled by the $\{h k l\}$ Miller plane notation, with wurtzite $\mathrm{ZnO}$ reflections shown by purple sticks and zincblende ZnS ones by brown sticks. (b) The $\{002\}:\{101\}$ peak intensity ratio from experimental patterns (black data points) and from simulations (red data points) plotted at the early stages of sulfidation. Lines are meant to guide the eye. (c) A magnified view of the experimental pXRD patterns plotted to highlight the peak reflection changes occurring in the early stages of sulfidation: $0,0.4,0.79,1.99$, and $2.78 \mu \mathrm{mol}$ of $\mathrm{S}^{2-}$. The color legend is the same as that of (a). (d) Simulated pXRD patterns for NCs at early stages of sulfidation, plotted with the same magnified view as that in (c). The legend specifies the molar ratio of S/Zn used in the pXRD simulation. The crystallographic parameters used for the pXRD simulations at each stage are tabulated in Table 2 . All patterns are shown vertically stacked and major reflections are labeled by the $\{h k l\}$ Miller plane notation.

gradually weaker in relative intensity, until they were fully extinguished. The final pXRD pattern is indicative of NPs in a single $\mathrm{ZnS}$ phase, as also confirmed by the elemental analysis (Table 1). The final ZnS NPs appear to have a broader size dispersion (Fig. 1d, standard deviation of $22 \%$ ) as compared to that of the initial ZnO NPs (Fig. 1b, standard deviation of 11\%). The final NPs are formed by sulfur-doping of ZnO NPs followed by nucleation and growth of separate $\mathrm{ZnS}$ domains, a process expected to be replete with inherent heterogeneities.

\section{Conclusion}

The scheme in Fig. 3 summarizes the mechanism of sulfidation that is drawn from the results taken as a whole. The sulfidation

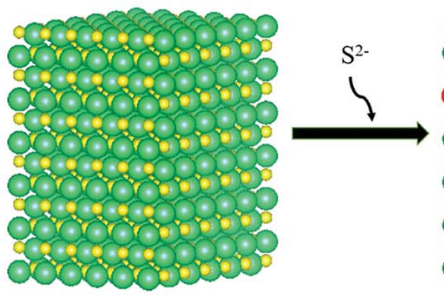

$\mathrm{W} \mathrm{ZnO}$

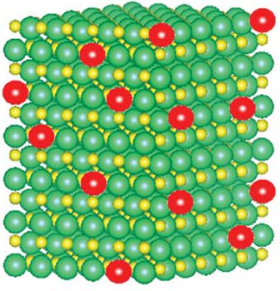

Sulfur-doped $\mathrm{ZnO}$

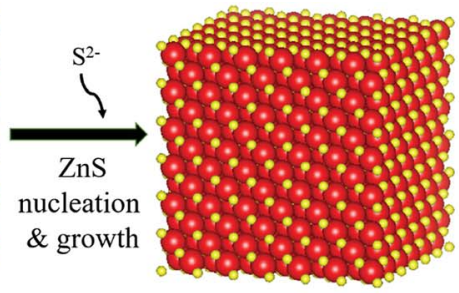

ZB ZnS

Fig. 3 Pictorial depiction of the atomistic mechanism of sulfidation. $Z n$ atoms are shown in yellow, $O$ in green, and $\mathrm{S}$ in red. The structures were generated in Vesta software. The ZnO crystallite was based on a wurtzite (W) P63mmc unit cell containing 2592 atoms. The sulfur-doped crystallite was made by replacing some of the $O$ atoms by $S$ atoms. The $Z n S$ crystallite is based on zincblende (ZB) F43m structure containing 1963 atoms. The morphologies and shapes of the final ZnS NPs formed by the nucleation-growth mechanism do not bear a topotaxial relationship with the initial ZnO NPs. 
of ZnO NPs is preceded by a stage where the NPs undergo substitutional doping by $\mathrm{S}^{2-}$. The wurtzite $\mathrm{ZnO}$ lattice remains otherwise unmodified in its structure. Above a concentration of ca. $30 \mathrm{~mol} \% \mathrm{~S}^{2-}$ dopants, $\mathrm{ZnS}$ nucleates from the $\mathrm{ZnO}$. The threshold concentration probably corresponds to the solubility limit of $\mathrm{S}^{2-}$ in $\mathrm{ZnO}$. As more $\mathrm{S}^{2-}$ becomes available, $\mathrm{ZnS}$ domains grow by supply of $\mathrm{Zn}^{2+}$ from $\mathrm{ZnO}$. The $\mathrm{O}^{2-}$ diffuses into solution. Thus, the complete phase transformation of the NPs from ZnO to ZnS cannot be considered simply as a topotactic exchange of anions in analogy to cation exchange reactions. ${ }^{36-47}$ Rather the post-doping stage sulfidation is more akin to a dissolution-reprecipitation process known in mineralogy, ${ }^{48}$ although the reprecipitation (nucleation-growth of $\mathrm{ZnS}$ ) may occur in interfacial contact with the sulfur-doped ZnO NPs. The ZnS NPs formed from the nucleation-growth process share neither the crystallographic structure nor the morphology or size of the initial ZnO NPs. In topotactic sulfidation, the differences between the equilibrium unit cells of $\mathrm{ZnO}$ and $\mathrm{ZnS}$ would result in lattice strain and stress; in sulfidation occurring via the reprecipitation-like mechanism found here, such stresses and any resulting thermodynamic or kinetic bottlenecks in the solid-state transformation would be relieved. This mechanism of sulfidation must be taken into consideration when developing small ZnO NP-based sorbents for uptake of sulfur compounds from fuels. The major restructuring involved in going from the oxide to the sulfide can have major implications for the engineering design and operation of NP-based sorbent columns.

\section{Conflicts of interest}

P. K. J. is an inventor on two patents on nanostructured $\mathrm{ZnO}$ sorbents and has served in the past as a scientific consultant on nanostructured $\mathrm{ZnO}$ for desulfurization technologies.

\section{Acknowledgements}

P. B. conducted materials synthesis and characterization, performed simulations and analysis, and co-wrote the manuscript. P. K. J. conceived the project, designed experiments, performed supporting simulations and analysis, and wrote the manuscript. We acknowledge the donors of the American Chemical Society Petroleum Research Fund for support of this research. Ryan Sanders and Cody Tripp are acknowledged for their preliminary studies. This work was conducted in part at the Frederick Seitz Materials Research Laboratory.

\section{Notes and references}

1 A. Kołodziejczak-Radzimska and T. Jesionowski, Zinc Oxide-From Synthesis to Application: A Review, Materials, 2014, 7(4), 2833-2881.

2 A. Moezzi, A. M. McDonagh and M. B. Cortie, Zinc Oxide Particles: Synthesis, Properties and Applications, Chem. Eng. J., 2012, 185-186, 1-22.

3 X. Tang, E. S. G. Choo, L. Li, J. Ding and J. Xue, Synthesis of ZnO Nanoparticles with Tunable Emission Colors and Their
Cell Labeling Applications, Chem. Mater., 2010, 22(11), 33833388.

4 K. Ghule, A. V. Ghule, B.-J. Chen and Y.-C. Ling, Preparation and Characterization of ZnO Nanoparticles Coated Paper and Its Antibacterial Activity Study, Green Chem., 2006, 8(12), 1034-1041.

5 R. Tankhiwale and S. K. Bajpai, Preparation, Characterization and Antibacterial Applications of $\mathrm{ZnO}$ Nanoparticles Coated Polyethylene Films for Food Packaging, Colloids Surf., B, 2012, 90, 16-20.

6 B. Stieberova, M. Zilka, M. Ticha, F. Freiberg, P. CaramazanaGonzález, J. McKechnie and E. Lester, Application of ZnO Nanoparticles in a Self-Cleaning Coating on a Metal Panel: An Assessment of Environmental Benefits, ACS Sustainable Chem. Eng., 2017, 5(3), 2493-2500.

7 A. M. El Saeed, M. A. El-Fattah and A. M. Azzam, Synthesis of ZnO Nanoparticles and Studying Its Influence on the Antimicrobial, Anticorrosion and Mechanical Behavior of Polyurethane Composite for Surface Coating, Dyes Pigm., 2015, 121, 282-289.

8 J. Ma, W. Zhu, Y. Tian and Z. Wang, Preparation of Zinc Oxide-Starch Nanocomposite and Its Application on Coating, Nanoscale Res. Lett., 2016, 11(1), 200.

9 D. Álvarez, A. Collazo, X. R. Nóvoa and C. Pérez, Assessment of ZnO Nanoparticles as Anticorrosive Pigment in Hybrid Sol-Gel Films, Prog. Org. Coat., 2016, 96, 3-12.

10 T. G. Smijs and S. Pavel, Titanium Dioxide and Zinc Oxide Nanoparticles in Sunscreens: Focus on Their Safety and Effectiveness, Nanotechnol., Sci. Appl., 2011, 4, 95-112.

11 J. Yin, Q. Lu, Z. Yu, J. Wang, H. Pang and F. Gao, Hierarchical ZnO Nanorod-Assembled Hollow Superstructures for Catalytic and Photoluminescence Applications, Cryst. Growth Des., 2010, 10(1), 40-43.

12 J. Zhang, Y. Wang, S. Zheng, H. Xue and H. Pang, N-Doped Mesoporous ZnO with Oxygen Vacancies for Stable Hydrazine Electrocatalysis, ChemNanoMat, 2018, 4, DOI: 10.1002/cnma.201800363.

13 H. Pang, Y. Ma, G. Li, J. Chen, J. Zhang, H. Zheng and W. Du, Facile Synthesis of Porous ZnO-NiO Composite Micropolyhedrons and Their Application for High Power Supercapacitor Electrode Materials, Dalton Trans., 2012, 41(43), 13284-13291.

14 J. Zhang, P. Gu, J. Xu, H. Xue and H. Pang, High Performance of Electrochemical Lithium Storage Batteries: ZnO-Based Nanomaterials for Lithium-Ion and Lithium-Sulfur Batteries, Nanoscale, 2016, 8(44), 18578-18595.

15 A. A. Keller, S. McFerran, A. Lazareva and S. Suh, Global Life Cycle Releases of Engineered Nanomaterials, J. Nanopart. Res., 2013, 15(6), 1692.

16 V. Kandavelu, H. Kastien and K. R. Thampi, Photocatalytic Degradation of Isothiazolin-3-Ones in Water and Emulsion Paints Containing Nanocrystalline $\mathrm{TiO}_{2}$ and $\mathrm{ZnO}$ Catalysts, Appl. Catal., B, 2004, 48(2), 101-111.

17 B. Nowack and T. D. Bucheli, Occurrence, Behavior and Effects of Nanoparticles in the Environment, Environ. Pollut., 2007, 150(1), 5-22. 
18 R. B. Reed, D. A. Ladner, C. P. Higgins, P. Westerhoff and J. F. Ranville, Solubility of Nano-Zinc Oxide in Environmentally and Biologically Important Matrices, Environ. Toxicol. Chem., 2012, 31(1), 93-99.

19 L. J. Gimbert, R. E. Hamon, P. S. Casey and P. J. Worsfold, Partitioning and Stability of Engineered ZnO Nanoparticles in Soil Suspensions Using Flow Field-Flow Fractionation, Environ. Chem., 2007, 4(1), 8-10.

20 O. Bondarenko, K. Juganson, A. Ivask, K. Kasemets, M. Mortimer and A. Kahru, Toxicity of Ag, CuO and $\mathrm{ZnO}$ Nanoparticles to Selected Environmentally Relevant Test Organisms and Mammalian Cells in vitro: A Critical Review, Arch. Toxicol., 2013, 87(7), 1181-1200.

21 Y. Ju-Nam and J. R. Lead, Manufactured Nanoparticles: An Overview of Their Chemistry, Interactions and Potential Environmental Implications, Sci. Total Environ., 2008, 400(1), 396-414.

22 F. Gottschalk, T. Sonderer, R. W. Scholz and B. Nowack, Modeled Environmental Concentrations of Engineered Nanomaterials $\left(\mathrm{TiO}_{2}, \mathrm{ZnO}, \mathrm{Ag}, \mathrm{CNT}\right.$, Fullerenes) for Different Regions, Environ. Sci. Technol., 2009, 43(24), 9216-9222.

23 M. Behl, J. Yeom, Q. Lineberry, P. K. Jain and M. A. Shannon, A Regenerable Oxide-Based $\mathrm{H}_{2} \mathrm{~S}$ Adsorbent with Nanofibrous Morphology, Nat. Nanotechnol., 2012, 7(12), 810-815.

$24 \mathrm{M}$. Behl and P. K. Jain, Catalytic Activation of a Solid Oxide in Electronic Contact With Gold Nanoparticles, Angew. Chem. Int. Ed., 2015, 54(3), 992-997.

25 M. A. Sayyadnejad, H. R. Ghaffarian and M. Saeidi, Removal of Hydrogen Sulfide by Zinc Oxide Nanoparticles in Drilling Fluid, Int. J. Environ. Sci. Technol., 2008, 5(4), 565-569.

26 X. Wang, T. Sun, J. Yang, L. Zhao and J. Jia, Low-Temperature $\mathrm{H}_{2} \mathrm{~S}$ Removal from Gas Streams with SBA-15 Supported ZnO Nanoparticles, Chem. Eng. J., 2008, 142(1), 48-55.

27 J. Skrzypski, I. Bezverkhyy, O. Heintz and J.-P. Bellat, Low Temperature $\mathrm{H}_{2} \mathrm{~S}$ Removal with Metal-Doped Nanostructure ZnO Sorbents: Study of the Origin of Enhanced Reactivity in Cu-Containing Materials, Ind. Eng. Chem. Res., 2011, 50(9), 5714-5722.

28 I. Rosso, C. Galletti, M. Bizzi, G. Saracco and V. Specchia, Zinc Oxide Sorbents for the Removal of Hydrogen Sulfide from Syngas, Ind. Eng. Chem. Res., 2003, 42(8), 1688-1697.

29 S. Lew, K. Jothimurugesan and M. Flytzani-Stephanopoulos, High-Temperature Hydrogen Sulfide Removal from Fuel Gases by Regenerable Zinc Oxide-Titanium Dioxide Sorbents, Ind. Eng. Chem. Res., 1989, 28(5), 535-541.

30 J. Park, H. Zheng, Y. Jun and A. P. Alivisatos, Hetero-Epitaxial Anion Exchange Yields Single-Crystalline Hollow Nanoparticles, J. Am. Chem. Soc., 2009, 131(39), 1394313945.

31 V. Subramanian, E. E. Wolf and P. V. Kamat, Green Emission to Probe Photoinduced Charging Events in $\mathrm{ZnO}-\mathrm{Au}$ Nanoparticles. Charge Distribution and Fermi-Level Equilibration, J. Phys. Chem. B, 2003, 107(30), 7479-7485.

32 H. Karzel, W. Potzel, M. Köfferlein, W. Schiessl, M. Steiner, U. Hiller, G. M. Kalvius, D. W. Mitchell, T. P. Das, P. Blaha, et al., Lattice Dynamics and Hyperfine Interactions in $\mathrm{ZnO}$ and ZnSe at High External Pressures, Phys. Rev. B, 1996, 53(17), 11425-11438.

33 H. Morkoç and Ü. Özgür, General Properties of ZnO, in Zinc Oxide, Wiley-VCH Verlag GmbH \& Co. KGaA, 2009; pp. 1-76.

34 B. K. Meyer, A. Polity, B. Farangis, Y. He, D. Hasselkamp, T. Krämer and C. Wang, Structural Properties and Bandgap Bowing of ZnO1-xSx Thin Films Deposited by Reactive Sputtering, Appl. Phys. Lett., 2004, 85(21), 49294931.

35 X. Fang, T. Zhai, U. K. Gautam, L. Li, L. Wu, Y. Bando and D. Golberg, ZnS Nanostructures: From Synthesis to Applications, Prog. Mater. Sci., 2011, 56(2), 175-287.

36 D. H. Son, S. M. Hughes, Y. Yin and A. Paul Alivisatos, Cation Exchange Reactions in Ionic Nanocrystals, Science, 2004, 306(5698), 1009-1012.

37 J. B. Rivest and P. K. Jain, Cation Exchange on the Nanoscale: An Emerging Technique for New Material Synthesis, Device Fabrication, and Chemical Sensing, Chem. Soc. Rev., 2013, 42(1), 89-96.

38 B. J. Beberwyck and A. P. Alivisatos, Ion Exchange Synthesis of III-V Nanocrystals, J. Am. Chem. Soc., 2012, 134(49), 19977-19980.

39 S. L. White, P. Banerjee, I. Chakraborty and P. K. Jain, Ion Exchange Transformation of Magic-Sized Clusters, Chem. Mater., 2016, 28(22), 8391-8398.

40 P. K. Jain, L. Amirav, S. Aloni and A. P. Alivisatos, Nanoheterostructure Cation Exchange: Anionic Framework Conservation, J. Am. Chem. Soc., 2010, 132(29), 9997-9999.

41 R. D. Robinson, B. Sadtler, D. O. Demchenko, C. K. Erdonmez, L.-W. Wang and A. P. Alivisatos, Spontaneous Superlattice Formation in Nanorods Through Partial Cation Exchange, Science, 2007, 317(5836), 355-358.

42 P. K. Jain, K. Manthiram, J. H. Engel, S. L. White, J. A. Faucheaux and A. P. Alivisatos, Doped Nanocrystals as Plasmonic Probes of Redox Chemistry, Angew. Chem., Int. Ed., 2013, 52(51), 13671-13675.

43 M. V. Kovalenko, L. Manna, A. Cabot, Z. Hens, D. V. Talapin, C. R. Kagan, V. I. Klimov, A. L. Rogach, P. Reiss, D. J. Milliron, et al., Prospects of Nanoscience with Nanocrystals, ACS Nano, 2015, 9(2), 1012-1057.

44 H. Li, M. Zanella, A. Genovese, M. Povia, A. Falqui, C. Giannini and L. Manna, Sequential Cation Exchange in Nanocrystals: Preservation of Crystal Phase and Formation of Metastable Phases, Nano Lett., 2011, 11(11), 4964-4970.

45 D. Dumett Torres, P. Banerjee, S. Pamidighantam and P. K. Jain, A Non-Natural Wurtzite Polymorph of HgSe: A Potential 3D Topological Insulator, Chem. Mater., 2017, 29(15), 6356-6366.

46 P. Banerjee and P. K. Jain, Lithiation of Copper Selenide Nanocrystals, Angew. Chem. Int. Ed., 2018, 57(30), 9315-9319.

47 S. L. White, P. Banerjee and P. K. Jain, Liquid-like Cationic Sub-Lattice in Copper Selenide Clusters, Nat. Commun., 2017, 8, 14514.

48 A. Putnis, Mineral Replacement Reactions: From Macroscopic Observations to Microscopic Mechanisms, Mineral. Mag., 2002, 66(5), 689-708. 